Georgian Mathematical Journal

Volume 13 (2006), Number 2, 199-214

\title{
SEMIMARTINGALE LOCAL TIME AND THE AMERICAN PUT OPTION
}

\author{
PETRE BABILUA
}

\begin{abstract}
A new result is obtained on the vanishing of the local time of a non-negative continuous semimartingale at zero. Based on this result, an early exercise premium representation of a value function of the American put option is obtained in a one-dimensional general diffusion model.
\end{abstract}

2000 Mathematical Subject Classification: 60G48, 60G40, 91B24.

Key words and phrases: American put option, Snell envelope, local time, value process, optimal stopping.

In this paper we investigate the American put option problem in a general diffusion model and its related optimal stopping problem. The main result of the investigation is the representation of a value function of the American put option as a sum of the corresponding value function of the European type put option and the early exercise premium. In the finance literature this representation is known under the name of the early exercise premium. It was derived by many authors in terms of the well known Black-Scholes model (i.e., when the dynamics of share values is described by geometric Brownian motion). Among them were N. El Karoui and I. Karatzas [5] and S. Jacka [3]. The former authors proceeded from the results of the theory of balayage of general type random processes, while Jacka used the fact that a value function of the American put option is a solution of the problem with a free boundary (Stefan's problem). A thorough investigation of the American put option problem in the BlackScholes model was carried out by $\mathrm{R}$. Myneni [8]. It should be however noted that the proofs of many authors somewhat lack the clearness.

Our approach is based on studying the properties of local times of general type continuous semimartingales and, as a result, we obtain a sufficient condition (Theorem 3) for the vanishing of local times at zero of non-negative continuous semimartingales. The verification of this condition for the American put option problem has turned out to be a simple matter. In particular, using this condition we derive an early exercise premium representation (Theorem 4) for a value function of the American put option in terms of a general one-dimensional diffusion model. The proof we give is quite clear. Furthermore, our result allows one to directly obtain as a particular case the early exercise premium representation for a value function of the American put option in terms of the well-known Black-Scholes model.

Let $(\Omega, \mathcal{F}, \mathrm{P})$ be a probability space with a standard Wiener process $\left(W_{t}, \mathcal{F}_{t}^{W}\right)$, $0 \leq t \leq T$, defined on it. We will consider on $(\Omega, \mathcal{F}, \mathrm{P})$ the finance market with two assets $\left(B_{t}, S_{t}\right), 0 \leq t \leq T$, where $B_{t}$ is the bank account value at time $t$ and 
$S_{t}$ is the share price at time $t$. The evolution of these assets obeys the equations

$$
\begin{gathered}
d B_{t}=r(t) B_{t} d t, \quad 0 \leq t \leq T, \quad B_{0}=1 \\
d S_{t}=r(t) S_{t} d t+\sigma\left(t, S_{t}\right) S_{t} d W_{t}, \quad 0 \leq t \leq T, \quad S_{0}>0
\end{gathered}
$$

where the continuous functions $r(t), \widetilde{\sigma}(t, x)=\sigma(t, x) x, 0 \leq t \leq T, x>0$, are assumed to be such that

1) $0 \leq r(t) \leq R$

2) $0<\bar{\sigma} \leq \sigma(t, x) \leq C$,

3) $|\widetilde{\sigma}(t, x)-\tilde{\sigma}(t, y)| \leq K|x-y|$.

Note that the random process $\frac{S_{t}}{B_{t}}$ is a martingale with respect to filtration $\mathcal{F}_{t}^{W}$.

Conditions (2) guarantee the existence of a unique strong solution of (1) and also of the following stochastic equation with its initial condition:

$$
\begin{aligned}
d S_{u}(t, x) & =r(u) S_{u}(t, x) d u \\
& +\sigma\left(u, S_{u}(t, x)\right) S_{u}(t, x) d W_{u}, \quad t \leq u \leq T, \\
S_{t}(t, x) & =x, \quad x>0 .
\end{aligned}
$$

Let us consider the American put option for the share $S_{t}$ with the payoff function $g(x)=(c-x)^{+}$. The corresponding payoff process $\widetilde{X}_{t}$ has the form $\widetilde{X}_{t}=g\left(S_{t}\right)$, while the value process $V_{t}$ of the American option is written as

$$
V_{t}=\operatorname{essipup}_{t \leq \tau \leq T} \mathrm{E}\left(e^{-\int_{t}^{\tau} r(u) d u} g\left(S_{\tau}\right) \mid \mathcal{F}_{t}^{W}\right), \quad 0 \leq t \leq T .
$$

It is clear that $V_{t}=B_{t} Y_{t}$, where

$$
B_{t}=e^{\int_{0}^{t} r(u) d u}, \quad Y_{t}=\underset{t \leq \tau \leq T}{\operatorname{essipup}} \mathrm{E}\left(e^{-\int_{0}^{\tau} r(u) d u} g\left(S_{\tau}\right) \mid \mathcal{F}_{t}^{W}\right) .
$$

Note that the value process $V_{t}$ of the American put option majorizes the payoff process $g\left(S_{t}\right), V_{t} \geq g\left(S_{t}\right), 0 \leq t \leq T$, and $Y_{t} \geq X_{t}$, where

$$
X_{t}=e^{-\int_{0}^{t} r(u) d u} g\left(S_{t}\right), \quad 0 \leq t \leq T
$$

Here $X_{t}$ is the discounted payoff process while the process $Y_{t}$ is its Snell envelope (a minimal supermartingale that majorizes $X_{t}$ ).

Along with the value process of the American put option, we define the value function of this option

$$
v^{T}(t, x)=\sup _{t \leq \tau \leq T} \mathrm{E}\left(e^{-\int_{t}^{\tau} r(u) d u}\left(c-S_{\tau}(t, x)\right)^{+}\right), \quad 0 \leq t \leq T, x>0 .
$$

It is obvious that it is also a value function of the corresponding optimal stopping problem of the nonhomogeneous Markov diffusion process $S_{t}$.

Now let us introduce the following notation:

$$
D^{T} \equiv\left\{(t, x): \quad 0 \leq t<T, \quad 0<x, \quad v^{T}(t, x)>g(x)\right\}
$$


where $D^{T}$ is the continuation domain of stopping problem; $D_{t}^{T}$ is the section of $D^{T}$ at a point $t, 0 \leq t<T$,

$$
D_{t}^{T} \equiv\left\{x:(t, x) \in D^{T}\right\} .
$$

We denote by $P(t, x ; u, B), u \geq t, x>0$, with a Borel subset $B$ of $(0,+\infty)$ the transition probability of the nonhomogeneous diffusion process $S_{t}$.

As is known (see [1], p. 445, formula (10)), for the random processes $X_{t}$ and $Y_{t}$ the following expansions are valid:

$$
\begin{gathered}
X_{t}=X_{0}+m_{t}+A_{t}, \quad A_{t}=A_{t}^{+}-A_{t}^{-}, \quad 0 \leq t \leq T, \\
Y_{t}=Y_{0}+M_{t}-C_{t}, \quad 0 \leq t \leq T,
\end{gathered}
$$

where $m_{t}, M_{t}$ are uniformly integrable martingales, while the processes $A_{t}^{+}, A_{t}^{-}$, $C_{t}$ are predictable nondecreasing integrable processes, $A_{0}^{+}=A_{0}^{-}=C_{0}=0$.

Let us now prove the lemma which will be used in proving our next theorem.

Lemma 1. Consider the process $S_{u}(t, x)$ satisfying equation (3). If $0<x<$ $y$, then

$$
S_{u}(t, x) \leq S_{u}(t, y) \text { P-a.s. for all } u, t \leq u \leq T .
$$

Proof. It is clear that the inequality $S_{u}(t, x) \leq S_{u}(t, y)$ P-a.s. is equivalent to the equality $\left(S_{u}(t, x)-S_{u}(t, y)\right)^{+}=0$ P-a.s., which in turn is the same as

$$
\mathrm{E}\left(S_{u}(t, x)-S_{u}(t, y)\right)^{+}=0 .
$$

Due to (3) we have

$$
\begin{aligned}
d\left(S_{u}(t, x)-S_{u}(t, y)\right) & =r(u)\left(S_{u}(t, x)-S_{u}(t, y)\right) d u \\
+ & \left(\widetilde{\sigma}\left(u, S_{u}(t, x)\right)-\widetilde{\sigma}\left(u, S_{u}(t, y)\right)\right) d W_{u}, \quad t \leq u \leq T .
\end{aligned}
$$

Let us write the well-known Tanaka-Meyer formula (see, e.g., [9]) for the process $\left(S_{u}(t, x)-S_{u}(t, y)\right)^{+}$

$$
\begin{gathered}
\left(S_{u}(t, x)-S_{u}(t, y)\right)^{+}=(x-y)^{+} \\
+\int_{t}^{u} I_{\left(S_{v}(t, x)-S_{v}(t, y)>0\right)} d\left(S_{v}(t, x)-S_{v}(t, y)\right)+\frac{1}{2} L_{u}^{\circ}(S(t, x)-S(t, y)) \\
=\int_{t}^{u} I_{\left(S_{v}(t, x)-S_{v}(t, y)>0\right)} r(v)\left(S_{v}(t, x)-S_{v}(t, y)\right) d v \\
+\int_{t}^{u} I_{\left(S_{v}(t, x)-S_{v}(t, y)>0\right)}\left(\widetilde{\sigma}\left(v, S_{v}(t, x)\right)-\widetilde{\sigma}\left(v, S_{v}(t, y)\right)\right) d W_{v} \\
+\frac{1}{2} L_{u}^{\circ}(S(t, x)-S(t, y)) .
\end{gathered}
$$

Since in (9) the last stochastic integral is a square integrable martingale, its mathematical expectation is equal to zero (see [7], Ch. IV, §2). 
Taking the mathematical expectation of both sides of equality (9), we obtain

$$
\begin{gathered}
\mathrm{E}\left(S_{u}(t, x)-S_{u}(t, y)\right)^{+} \\
=\mathrm{E} \int_{t}^{u} r(v)\left(S_{v}(t, x)-S_{v}(t, y)\right)^{+} d v+\frac{1}{2} \mathrm{E} L_{u}^{\circ}(S(t, x)-S(t, y)) .
\end{gathered}
$$

Using Yor's corollary [13], we have

$$
\begin{gathered}
L_{u}^{\circ}(S(t, x)-S(t, y)) \\
=\lim _{\varepsilon \rightarrow 0} \frac{1}{\varepsilon} \int_{t}^{u} I_{\left(0<S_{v}(t, x)-S_{v}(t, y) \leq \varepsilon\right)}\left(\widetilde{\sigma}\left(v, S_{v}(t, x)\right)-\widetilde{\sigma}\left(v, S_{v}(t, y)\right)\right)^{2} d v \\
\leq \varlimsup_{\varepsilon \rightarrow 0} \frac{1}{\varepsilon} \int_{t}^{u} I_{\left(0<S_{v}(t, x)-S_{v}(t, y) \leq \varepsilon\right)} K^{2}\left(S_{v}(t, x)-S_{v}(t, y)\right)^{2} d v \leq \varlimsup_{\varepsilon \rightarrow 0} \int_{t}^{u} K^{2} \varepsilon d v=0,
\end{gathered}
$$

which implies

$$
\mathrm{E}\left(S_{u}(t, x)-S_{u}(t, y)\right)^{+}=\mathrm{E} \int_{t}^{u} r(v)\left(S_{v}(t, x)-S_{v}(t, y)\right)^{+} d v
$$

Denoting

we obtain

$$
\varphi(u) \equiv \mathrm{E}\left(S_{u}(t, x)-S_{u}(t, y)\right)^{+}, \quad t \leq u \leq T
$$

$$
\varphi(u) \leq R \int_{t}^{u} \varphi(v) d v
$$

for all $u$ and using Gronwall's lemma, we have

$$
\mathrm{E}\left(S_{u}(t, x)-S_{u}(t, y)\right)^{+}=0 \text {. }
$$

Theorem 1. The section of $D^{T}$ at the point $t$ has the form

$$
D_{t}^{T}=\left(b^{T}(t),+\infty\right), \quad 0 \leq t<T,
$$

where $b^{T}(t)$ is some function which satisfies the condition

$$
0<b^{T}(t)<c, \quad 0 \leq t<T .
$$

Proof. It is clear that $0 \notin D_{t}^{T}$. Indeed, suppose that $0 \in D_{t}^{T}$, it is the same as $\left\{(t, 0) \in D^{T}: v^{T}(t, 0)>g(0)=c\right\}$, which contradicts the condition $v^{T}(t, x)<c$.

We have

$$
\begin{aligned}
d S_{u}(t, x) & =r(u) S_{u}(t, x) d u \\
& +\sigma\left(u, S_{u}(t, x)\right) S_{u}(t, x) d W_{u}, \quad t \leq u \leq T, \\
S_{t}(t, x) & =x, \quad x>0, \\
d S_{u}(t, y) & =r(u) S_{u}(t, y) d u \\
& +\sigma\left(u, S_{u}(t, y)\right) S_{u}(t, y) d W_{u}, \quad t \leq u \leq T,
\end{aligned}
$$




$$
S_{t}(t, y)=y, \quad y>0 \text {. }
$$

We are to prove that if $x \in D_{t}^{T}$ and $y>0$, then $y \in D_{t}^{T}$.

Note that for the stopping problem for the diffusion process $S_{u}(t, x)$ there exists an optimal stopping time $\tau^{*}(t, x)$ (see [6], Theorems 3.1.8-3.1.10)

Using the preceding lemma, for the value function $v^{T}(t, x)$ defined in $(6)$ we have

$$
\begin{aligned}
& v^{T}(t, y)-v^{T}(t, x)=v^{T}(t, y)-\mathrm{E}\left[e^{-\tau_{t}^{\tau^{*}(t, x)} r(u) d u}\left(c-S_{\tau^{*}(t, x)}(t, x)\right)^{+}\right] \\
& \geq \mathrm{E}\left[e^{-\int_{t}^{\tau^{*}(t, x)} r(u) d u}\left(c-S_{\tau^{*}(t, x)}(t, y)\right)^{+}-e^{-\int_{t}^{\tau^{*}(t, x)} r(u) d u}\left(c-S_{\tau^{*}(t, x)}(t, x)\right)^{+}\right] \\
& =\mathrm{E}\left[e^{-\int_{t}^{\tau^{*}(t, x)} r(u) d u}\left(c-S_{\tau^{*}(t, x)}(t, y)\right)-e^{-\int_{t}^{\tau^{*}(t, x)} r(u) d u}\left(c-S_{\tau^{*}(t, x)}(t, x)\right)\right] \\
& +\mathrm{E}\left[e^{-\int_{t}^{\tau^{*}(t, x)} r(u) d u}\left(c-S_{\tau^{*}(t, x)}(t, y)\right)^{-}-e^{-\int_{t}^{\tau^{*}(t, x)} r(u) d u}\left(c-S_{\tau^{*}(t, x)}(t, x)\right)^{-}\right] \\
& \geq \mathrm{E}\left[e^{-\int_{t}^{\tau^{*}(t, x)} r(u) d u}\left(c-S_{\tau^{*}(t, x)}(t, y)\right)-e^{-\int_{t}^{\tau^{*}(t, x)} r(u) d u}\left(c-S_{\tau^{*}(t, x)}(t, x)\right)\right] \\
& =\mathrm{E}\left[e^{-\tau^{\tau^{*}(t, x)} r(u) d u}\left(S_{\tau^{*}(t, x)}(t, x)-S_{\tau^{*}(t, x)}(t, y)\right)\right] \\
& =\mathrm{E}\left[S_{t}(t, x)-S_{t}(t, y)\right]=x-y,
\end{aligned}
$$

i.e., $v^{T}(t, y) \geq(x-y)+v^{T}(t, x)$. Since $v^{T}(t, x)>(c-x)^{+}$, we have

$$
v^{T}(t, y)>x-y+(c-x)^{+}>x-y+c-x=c-y,
$$

where $v^{T}(t, y)>0$, i.e., $v^{T}(t, y)>(c-y)^{+}$.

To derive the early exercise premium representation of the American put option, we need to investigate the properties of a local time for general continuous semimartingales. To this end, let us consider some continuous semimartingale $Z_{t}, 0 \leq t \leq T$, and its set of zeros

$$
H=\left\{(t, w): Z_{t}(w)=0\right\} .
$$

Denote by $L_{t}^{\circ}(Z)$ the right local time of the semimartingale at zero. We also need to use the following stopping times

$$
D_{t}(w)=\inf \left\{s>t: Z_{s}(w) \neq 0\right\} \wedge T, \quad 0 \leq t \leq T,
$$

and to consider the corresponding predictable random set

$$
\widetilde{H}_{0}=\bigcup_{r}\left(r, D_{r}\right]
$$

where $\left(r, D_{r}\right]$ denotes the stochastic intervals and the union is taken over all rationals $r$, such that $0 \leq r \leq T$. 
Theorem 2. Let us consider some continuous semimartingale $Z_{t}, 0 \leq t \leq T$, and its related set of zeros $H=\left\{(t, w): Z_{t}(w)=0\right\}$. Let us consider its arbitrary, progressively measurable open subset $H_{0} \subseteq H$. Then the following relations are valid:
I. $\quad \int_{0}^{t} I_{\widetilde{H}_{0}} d L_{s}^{\circ}(Z)=0$
$\int_{0}^{t} I_{\widetilde{H}_{0}} d L_{s}^{\circ}(-Z)=0$
II. $\quad \int_{0}^{t} I_{H_{0}} d L_{s}^{\circ}(Z)=0$
$\int_{0}^{t} I_{H_{0}} d L_{s}^{\circ}(-Z)=0$

In this case, if $H_{0}$ is also a predictable random set, then

$$
\text { III. } \quad \int_{0}^{t} I_{H_{0}} d Z_{s}(w)=0, \quad 0 \leq t \leq T .
$$

Proof. We have

$$
\begin{gathered}
Z_{t}=Z_{0}+\int_{0}^{t}\left(I_{\left(Z_{s}>0\right)}+I_{\left(Z_{s}=0\right)}+I_{\left(Z_{s}<0\right)}\right) d Z_{s}, \\
Z_{t}^{+}=Z_{0}^{+}+\int_{0}^{t}\left(I_{\left(Z_{s}>0\right)} d Z_{s}+\frac{1}{2} L_{t}^{\circ}(Z),\right. \\
\left(-Z_{t}\right)^{+}=\left(-Z_{0}\right)^{+}+\int_{0}^{t} I_{\left(-Z_{s}>0\right)} d\left(-Z_{s}\right)+\frac{1}{2} L_{t}^{\circ}(-Z) .
\end{gathered}
$$

From equality (15) we obtain

$$
Z_{t}^{-}=Z_{0}^{-}-\int_{0}^{t} I_{\left(Z_{s}<0\right)} d Z_{s}+\frac{1}{2} L_{t}^{\circ}(-Z)
$$

Subtracting (16) from (14) gives

$$
Z_{t}=Z_{0}+\int_{0}^{t}\left(I_{\left(Z_{s}>0\right)}+I_{\left(Z_{s}<0\right)}\right) d Z_{s}+\frac{1}{2}\left(L_{t}^{\circ}(Z)-L_{t}^{\circ}(-Z)\right) .
$$

If we equate equalities (17) and (13) to each other, then we have

$$
\int_{0}^{t} I_{\left(Z_{s}=0\right)} d Z_{s}=\frac{1}{2}\left(L_{t}^{\circ}(Z)-L_{t}^{\circ}(-Z)\right) .
$$


Consider the stochastic interval $\left(t, D_{t}(w)\right]$. Then, using the above equalities, we obtain

$$
\begin{aligned}
& Z_{D_{t}}^{+}-Z_{t}^{+}=\int_{t}^{D_{t}} I_{\left(Z_{s}>0\right)} d Z_{s}+\frac{1}{2}\left(L_{D_{t}}^{\circ}(Z)-L_{t}^{\circ}(Z)\right), \\
& Z_{D_{t}}^{-}-Z_{t}^{-}=-\int_{0}^{D_{t}} I_{\left(Z_{s}<0\right)} d Z_{s}+\frac{1}{2}\left(L_{D_{t}}^{\circ}(-Z)-L_{t}^{\circ}(-Z)\right) .
\end{aligned}
$$

The latter two equalities are the same as

$$
\begin{aligned}
& Z_{D_{t}}^{+}-Z_{t}^{+}=\int_{t}^{T} I_{\left(t<s \leq D_{t}, Z_{s}>0\right)} d Z_{s}+\frac{1}{2}\left(L_{D_{t}}^{\circ}(Z)-L_{t}^{\circ}(Z)\right), \\
& Z_{D_{t}}^{-}-Z_{t}^{-}=-\int_{t}^{T} I_{\left(t<s \leq D_{t}, Z_{s}<0\right)} d Z_{s}+\frac{1}{2}\left(L_{D_{t}}^{\circ}(-Z)-L_{t}^{\circ}(-Z)\right) .
\end{aligned}
$$

Let us consider the random variables

$$
Z_{D_{t}}^{+}-Z_{t}^{+}, \quad Z_{D_{t}}^{-}-Z_{t}^{-}, \quad I_{\left(t<s \leq D_{t}, Z_{s}>0\right)}, \quad I_{\left(t<s \leq D_{t}, Z_{s}<0\right)}
$$

and for each $\omega \in \Omega$ let us distinguish two cases:

1) $D_{t}(\omega)=t$

2) $D_{t}(\omega)>t$.

In case 1$)$ the above-given random variables are equal to zero. In case 2), we observe that if $s$ is such that $t<s \leq D_{t}(\omega)$, then $Z_{s}=0$ and thus, by the continuity of the process $Z_{t}$, we have $Z_{D_{t}}=0, Z_{t}=0$, which means that in case 2) also all four random variables are equal to zero. Hence it follows that

$$
L_{D_{t}}^{\circ}(Z)=L_{t}^{\circ}(Z), \quad L_{D_{t}}^{\circ}(-Z)=L_{t}^{\circ}(-Z)
$$

and therefore

$$
\int_{\left(r, D_{r}\right]} d L_{s}^{\circ}(Z)=0, \quad \int_{\left(r, D_{r}\right]} d L_{s}^{\circ}(-Z)=0,
$$

By the definition of $\widetilde{H}_{0}$,

$$
\int_{\widetilde{H}_{0}} d L_{s}^{\circ}(Z)=0, \quad \int_{\widetilde{H}_{0}} d L_{s}^{\circ}(-Z)=0
$$

which is the same as

$$
\int_{0}^{t} I_{\widetilde{H}_{0}} d L_{s}^{\circ}(Z)=0, \quad \int_{0}^{t} I_{\widetilde{H}_{0}} d L_{s}^{\circ}(-Z)=0 .
$$

The first part is thereby proved. 
Since $H_{0} \subseteq \bigcup_{r}\left(r, D_{r}\right]=\widetilde{H}_{0}$, the following relation is valid:

$$
\int_{0}^{t} I_{H_{0}} d L_{s}^{\circ}(Z)=0, \quad \int_{0}^{t} I_{H_{0}} d L_{s}^{\circ}(-Z)=0 .
$$

From (18) we obtain

$$
I_{\left(Z_{s}=0\right)} d Z_{s}=\frac{1}{2} d\left(L_{t}^{\circ}(Z)-L_{t}^{\circ}(-Z)\right) .
$$

When $H_{0}$ is predictable, we can write

$$
\begin{gathered}
I_{H_{0}} I_{\left(Z_{s}=0\right)} d Z_{s}=\frac{1}{2} I_{H_{0}} d\left(L_{t}^{\circ}(Z)-L_{t}^{\circ}(-Z)\right), \\
\int_{0}^{t} I_{H_{0}} d Z_{s}=\frac{1}{2} \int_{0}^{t} I_{H_{0}} d L_{s}^{\circ}(Z)-\frac{1}{2} \int_{0}^{t} I_{H_{0}} d L_{s}^{\circ}(-Z)=0 .
\end{gathered}
$$

Theorem 3. Let $Z_{t}$ be a continuous non-negative semimartingale with its decomposition

$$
Z_{t}=Z_{0}+M_{t}^{z}+G_{t}^{+}-G_{t}^{-}, \quad 0 \leq t \leq T
$$

where $M_{t}^{z}$ is a local martingale, $G=G_{t}^{+}-G_{t}^{-}$is a process of finite variation, and $G_{t}^{+}, G_{t}^{-}$are some nondecreasing processes.

If $\int_{0}^{T} I_{\left(H \backslash \widetilde{H}_{0}\right)} d G_{s}^{+}=0$, then $L_{t}^{\circ}(Z)=0$ and $\int_{0}^{t} I_{\left(Z_{s}=0\right)} d Z_{s}=0$.

Proof. It is clear that the right local time $L_{t}^{\circ}(-Z)=0, t \geq 0$.

We have

$$
\begin{gathered}
\frac{1}{2} L_{t}^{\circ}(Z)=\int_{0}^{t} I_{\left(Z_{s}=0\right)} d Z_{s} \\
=\int_{0}^{t} I_{\left(Z_{s}=0\right)} d M_{s}^{z}+\int_{0}^{t} I_{\left(Z_{s}=0\right)} d G_{s}^{+}-\int_{0}^{t} I_{\left(Z_{s}=0\right)} d G_{s}^{-} .
\end{gathered}
$$

Using occupation time formula (see, e.g., [9], p. 216, Corollary 1) we obtain

$$
\int_{0}^{t} I_{\left(Z_{s}=0\right)} d\left\langle M^{z}\right\rangle_{s}=0
$$

so we have

$$
\int_{0}^{t} I_{\left(Z_{s}=0\right)} d M_{s}^{z}=0
$$


Using (21), from (20) we obtain

$$
\frac{1}{2} L_{t}^{\circ}(Z)+\int_{0}^{t} I_{\left(Z_{s}=0\right)} d G_{s}^{-}=\int_{0}^{t} I_{\left(Z_{s}=0\right)} d G_{s}^{+},
$$

from which we conclude that the random processes $\frac{1}{2} L_{t}^{\circ}(Z)$ and $\int_{0}^{t} I_{\left(Z_{s}=0\right)} d G_{s}^{-}$ are absolutely continuous with respect to the process $\int_{0}^{t} I_{\left(Z_{s}=0\right)} d G_{s}^{+}$. Thus for these random processes we have the representations

$$
\begin{aligned}
\frac{1}{2} L_{t}^{\circ}(Z) & =\int_{0}^{t} \mu_{s}^{1} I_{\left(Z_{s}=0\right)} d G_{s}^{+}, \\
\int_{0}^{t} I_{\left(Z_{s}=0\right)} d G_{s}^{-} & =\int_{0}^{t} \mu_{s}^{2} I_{\left(Z_{s}=0\right)} d G_{s}^{+},
\end{aligned}
$$

where the processes $\mu_{s}^{1}$ and $\mu_{s}^{2}$ are both vary in $[0,1]$.

From equality (22) we obtain

$$
\frac{1}{2} d L_{t}^{\circ}(Z)=\mu_{t}^{1} I_{\left(Z_{t}=0\right)} d G_{t}^{+}
$$

By virtue of Theorem 2, $\int_{0}^{t} I_{\widetilde{H}_{0}} d L_{s}^{\circ}(Z)=0$ and therefore $L_{t}^{\circ}(Z)=$ $\int_{0}^{t} I_{H \backslash \widetilde{H}_{0}} d L_{s}^{\circ}(Z)$. Hence

$$
L_{t}^{\circ}(Z)=2 \int_{0}^{t} I_{\left(H \backslash \widetilde{H}_{0}\right)} \mu_{s}^{1} I_{\left(Z_{s}=0\right)} d G_{s}^{+} \leq 2 \int_{0}^{t} I_{\left(H \backslash \widetilde{H}_{0}\right)} d G_{s}^{+}=0
$$

and thus we obtain $L_{t}^{\circ}(Z)=0, \int_{0}^{t} I_{\left(Z_{s}=0\right)} d Z_{s}=0$.

Before formulating the main result of this paper on the early exercise premium representation of the American put option, we need to prove one more lemma.

Lemma 2. Consider a random process $X_{t}(s, x)=\ln S_{t}(s, x), s \leq t \leq T$, where $S_{t}(s, x)$ is the random process defined by equation (3). Then $X_{t}(s, x)$ satisfies the stochastic differential equation

$$
d X_{t}(s, x)=\widehat{\mu}\left(t, X_{t}(s, x)\right) d t+\widehat{\sigma}\left(t, X_{t}(s, x)\right) d W_{t}, \quad s \leq t \leq T,
$$

where

$$
\widehat{\mu}(t, x)=r(t)-\frac{1}{2} \sigma^{2}\left(t, e^{x}\right), \quad \widehat{\sigma}(t, x)=\sigma\left(t, e^{x}\right),
$$


and is its unique solution. Moreover, if $c(t), 0 \leq t \leq T$, is some measurable function, then the relations

$$
\mathrm{P}\left\{X_{t}(s, x)=c(t)\right\}=0
$$

are valid for all $t \geq s$.

Proof. By virtue of equality (3) we have and using Ito's formula for $X_{t}(s, x)=$ $\ln S_{t}(s, x), s \leq t \leq T$, we obtain

$$
d \ln S_{t}(s, x)=\left(r(t)-\frac{1}{2} \sigma^{2}\left(t, S_{t}(s, x)\right)\right) d t+\sigma\left(t, S_{t}(s, x)\right) d W_{t}, \quad s \leq t \leq T,
$$

by which come to the desirable result $(24)$, i.e., $X_{t}(s, x)$ satisfies equation $(24)$ and is its unique solution. Indeed, suppose equation (24) has two solutions $X_{t}^{1}(s, x)$ and $X_{t}^{2}(s, x)$. Then $e^{X_{t}^{1}(s, x)}$ and $e^{X_{t}^{2}(s, x)}$ are solutions of equation (3), but this equation has only one solution and therefore $X_{t}^{1}(s, x)=X_{t}^{2}(s, x)$ for almost all $s \geq t$. If we follow [12], Theorem 9.1.9, then we have

$$
\mathrm{P}\left\{X_{t}(s, x)=c(t)\right\}=\int_{\{c(t)\}} \widehat{P}(s, x ; t, y) d y=0,
$$

where $\widehat{P}(s, x ; t, y)$ is the transition density of the nonhomogeneous Markov diffusion process $X_{t}(s, x)$.

We will now formulate the theorem on representation of the value function of the American put option.

Theorem 4. Let $b^{T}(t)$ be the boundary function of the continuation domain defined in Theorem 1. Assume that it has left and right limits $b^{T}(t+), b^{T}(t-)$, $0<t<T$. Then for the value function of the American put option the following early exercise premium representation is valid

$$
\begin{aligned}
v^{T}(t, x) & =\mathrm{E}\left(e^{-\int_{t}^{T} r(u) d u}\left(c-S_{T}(t, x)\right)^{+}\right) \\
& +\int_{t}^{T} c r(u) e^{-\int_{t}^{u} r(s) d s} P\left(t, x ; u,\left(0, b^{T}(u)\right]\right) d u, \quad 0 \leq t \leq T, \quad x>0,
\end{aligned}
$$

where $P(t, x ; u, \cdot)$ is the transition probability for the nonhomogeneous Markov diffusion process $S_{t}(s, x)$.

Proof. Consider the random process defined by equality (5):

$$
X_{t}=e^{-\int_{0}^{t} r(u) d u}\left(c-S_{t}\right)^{+} .
$$

For the process $\left(c-S_{t}\right)^{+}$we write the Tanaka-Meyer formula

$$
\left(c-S_{t}\right)^{+}=\left(c-S_{0}\right)^{+}+\int_{0}^{t} I_{\left(c-S_{t}>0\right)} d\left(c-S_{t}\right)+\frac{1}{2} L_{t}^{\circ}\left(c-S_{t}\right),
$$


we have

$$
\begin{gathered}
d\left(e^{-\int_{0}^{t} r(u) d u}\left(c-S_{t}\right)^{+}\right)=-r(t) e^{-\int_{0}^{t} r(u) d u}\left(c-S_{t}\right)^{+} d t+e^{-\int_{0}^{t} r(u) d u} d\left(c-S_{t}\right)^{+} \\
=-r(t) e^{-\int_{0}^{t} r(u) d u} d t\left(c-S_{t}\right)^{+}+e^{-\int_{0}^{t} r(u) d u}\left(-I_{\left(S_{t}<c\right)} d S_{t}+\frac{1}{2} L_{t}^{\circ}\left(c-S_{t}\right)\right) \\
=-r(t) e^{-\int_{0}^{t} r(u) d u} d t\left(c-S_{t}\right)^{+}-e^{-\int_{0}^{t} r(u) d u} I_{\left(S_{t}<c\right)} d S_{t}+\frac{1}{2} e^{-\int_{0}^{t} r(u) d u} d L_{t}^{\circ}\left(c-S_{t}\right) .
\end{gathered}
$$

Now, using (1) we obtain

$$
\begin{gathered}
d\left(e^{-\int_{0}^{t} r(u) d u}\left(c-S_{t}\right)^{+}\right)=-r(t) e^{-\int_{0}^{t} r(u) d u} d t\left(c-S_{t}\right)^{+}-e^{-\int_{0}^{t} r(u) d u} I_{\left(S_{t}<c\right)} r(t) S_{t} d t \\
-e^{-\int_{0}^{t} r(u) d u} I_{\left(S_{t}<c\right)} \sigma\left(t, S_{t}\right) S_{t} d W_{t}+\frac{1}{2} e^{-\int_{0}^{t} r(u) d u} d L_{t}^{\circ}\left(c-S_{t}\right) \\
=-r(t) e^{-\int_{0}^{t} r(u) d u}\left(\left(c-S_{t}\right)^{+}+I_{\left(S_{t}<c\right)} S_{t}\right) d t \\
+\frac{1}{2} e^{-\int_{0}^{t} r(u) d u} d L_{t}^{\circ}\left(c-S_{t}\right)-e^{-\int_{0}^{t} r(u) d u} I_{\left(S_{t}<c\right)} \sigma\left(t, S_{t}\right) S_{t} d W_{t} .
\end{gathered}
$$

Note that $\left(c-S_{t}\right)^{+}+I_{\left(S_{t}<c\right)} S_{t}=c I_{\left(S_{t}<c\right)}$ and therefore we have

$$
\begin{aligned}
d X_{t} & =-r(t) e^{-\int_{0}^{t} r(u) d u} c I_{\left(S_{t}<c\right)} d t+\frac{1}{2} e^{-\int_{0}^{t} r(u) d u} d L_{t}^{\circ}\left(c-S_{t}\right) \\
& -e^{-\int_{0}^{t} r(u) d u} I_{\left(S_{t}<c\right)} \sigma\left(t, S_{t}\right) S_{t} d W_{t},
\end{aligned}
$$

i.e., for the process $X_{t}$ we have obtained the representation

$$
X_{t}=m_{t}+A_{t}^{+}-A_{t}^{-},
$$

where

$$
A_{t}^{-}=\int_{0}^{t} \operatorname{cr}(u) I_{\left(S_{u}<c\right)} e^{-\int_{0}^{u} r(s) d s} d u
$$

Let us consider the nonnegative process $Z_{t}=Y_{t}-X_{t}, 0 \leq t \leq T$, where $Y_{t}$ and $X_{t}$ are the processes defined by equalities (4) and (5), respectively. Using decompositions (7) and (8) for $Y_{t}$ and $X_{t}$, we find that

$$
Z_{t}=Z_{0}+M_{t}^{z}-C_{t}-A_{t}, \quad M_{t}^{z}=M_{t}-m_{t}, \quad A_{t}=A_{t}^{+}-A_{t}^{-}, \quad 0 \leq t \leq T,
$$

where $m_{t}$ and $M_{t}$ are uniformly integrable martingales, while $A_{t}^{+}, A_{t}^{-}$and $C_{t}$ are predictable nondecreasing integrable processes, $A_{0}^{+}=A_{0}^{-}=C_{0}=0$.

We have

$$
\frac{1}{2} L_{t}^{\circ}(Z)=\int_{0}^{t} I_{\left(Z_{s}=0\right)} d Z_{s}=\int_{0}^{t} I_{\left(Z_{s}=0\right)} d M_{s}^{z}-\int_{0}^{t} I_{\left(Z_{s}=0\right)} d C_{s}-\int_{0}^{t} I_{\left(Z_{s}=0\right)} d A_{s} .
$$


It is known that $\int_{0}^{t} I_{\left(Z_{s}=0\right)} d M_{s}^{z}=0$.

It is also known (see [10], Lemma 1 ) that $\int_{0}^{t} I_{\left(Z_{s}=0\right)} d C_{s}=C_{t}$. Therefore we have

$$
\frac{1}{2} L_{t}^{\circ}(Z)+C_{t}+\int_{0}^{t} I_{\left(Z_{s}=0\right)} d A_{s}^{+}=\int_{0}^{t} I_{\left(Z_{s}=0\right)} d A_{s}^{-} .
$$

Taking the latter equality into account, we conclude that $\frac{1}{2} L_{t}^{\circ}(Z), C_{t}$, and $\int_{0}^{t} I_{\left(Z_{s}=0\right)} d A_{s}^{+}$are absolutely continuous processes with respect to the process $t$ $\int_{0}^{t} I_{\left(Z_{s}=0\right)} d A_{s}^{-}$.

Suppose that

$$
\int_{0}^{T} I_{H \backslash \widetilde{H}_{0}} d A_{s}^{-}=0 \quad \text { P-a.s. }
$$

If instead of the component $G_{t}^{+}$used in Theorem 3, we take $A_{t}^{-}$, and in the role of the component $G_{t}^{-}$we take $C_{t}+A_{t}^{+}$, then using Theorem 3 we obtain

$$
\begin{gathered}
L_{t}^{\circ}(Z)=0, \quad \int_{0}^{t} I_{\left(Z_{s}=0\right)} d Z_{s}=0, \\
C_{t}=-\int_{0}^{t} I_{\left(Z_{s}=0\right)} d A_{s} .
\end{gathered}
$$

Let us now prove that (25) is valid. Besides of $H$ and $\widetilde{H}_{0}$ introduced above by relations (10) and (12), respectively, let us introduce the following sets:

$$
\begin{aligned}
\bar{H}_{0} & =\left\{(t, w): 0<t \leq T, S_{t}(w)-b^{T}(t-)<0\right\} \\
\underline{H}_{0} & =\left\{(t, w): 0<t \leq T, S_{t}(w)-b^{T}(t)<0\right\} \\
N & =\left\{(t, w): 0<t \leq T, b^{T}(t-)=b^{T}(t)\right\} \\
& =\left\{t: 0<t \leq T, b^{T}(t-)=b^{T}(t)\right\} \times \Omega .
\end{aligned}
$$

Note that

$$
H=\left\{(t, w): 0 \leq t \leq T, S_{t}(w) \leq b^{T}(t)\right\}
$$

We have the inclusions:

$$
\bar{H}_{0} \subseteq \widetilde{H}_{0}, \quad \bar{H}_{0} \subseteq H, \quad H \backslash \widetilde{H}_{0} \subseteq H \backslash \bar{H}_{0} .
$$


In view of the latter inclusion for the validity of (25) it is sufficient to verify that $\int_{0}^{T} I_{\left(H \backslash \bar{H}_{0}\right)} d A_{s}^{-}=0$ holds. To prove this relation let us first verify that $H \backslash \bar{H}_{0} \subseteq\left(H \backslash \underline{H}_{0}\right) \cup N^{c}$.

Indeed, $\bar{H}_{0} \cap N=\underline{H}_{0} \cap N$ and

$$
\begin{gathered}
H \backslash \bar{H}_{0} \subseteq H \backslash\left(\bar{H}_{0} \cap N\right) \\
=H \backslash\left(\underline{H}_{0} \cap N\right)=\left(H \backslash \underline{H}_{0}\right) \cup(H \backslash N) \subseteq\left(H \backslash \underline{H}_{0}\right) \cup N^{c} .
\end{gathered}
$$

Therefore we have

$$
\begin{gathered}
\int_{0}^{T} I_{H \backslash \bar{H}_{0}} d A_{s}^{-} \leq \int_{0}^{T} I_{\left(H \backslash \underline{H}_{0}\right) \cup N^{c}} d A_{s}^{-} \leq \int_{0}^{T} I_{\left(S_{s}(w)=b^{T}(s)\right)} d A_{s}^{-}+\int_{0}^{T} I_{\left(b^{T}(s-) \neq b^{T}(s)\right)} d A_{s}^{-} \\
=\int_{0}^{T} I_{\left(S_{u}(w)=b^{T}(u)\right)} c r(u) I_{\left(S_{u}<c\right)} e^{-\int_{0}^{u} r(s) d s} d u \leq c R \int_{0}^{T} I_{\left(S_{u}(w)=b^{T}(u)\right)} d u .
\end{gathered}
$$

By virtue of Lemma 2, for $c(t)=\ln b^{T}(t)$ we have $\mathrm{P}\left\{S_{u}=b^{T}(u)\right\}=0$, $\int_{0}^{T} I_{\left(H \backslash \bar{H}_{0}\right)} d A_{s}^{-}=0$, which in turn implies (25) and because of (26) we obtain

$$
\begin{aligned}
C_{t} & =-\int_{0}^{t} I_{\left(Z_{s}=0\right)} d A_{s}=-\int_{0}^{t} I_{\left(S_{u} \leq b^{T}(u)\right)} d A_{u} \\
& =-\int_{0}^{t} I_{\left(S_{u} \leq b^{T}(u)\right)} d A_{u}^{+}+\int_{0}^{t} I_{\left(S_{u} \leq b^{T}(u)\right)} d A_{u}^{-} \\
& =-\frac{1}{2} \int_{0}^{t} I_{\left(S_{u} \leq b^{T}(u)\right)} e^{-\int_{0}^{u} r(s) d s} I_{\left(S_{u}=c\right)} d L_{u}^{\circ}\left(c-S_{t}\right) \\
& +\int_{0}^{t} I_{\left(S_{u} \leq b^{T}(u)\right)} c r(u) e^{-\int_{0}^{u} r(s) d s} I_{\left(S_{u}<c\right)} d u .
\end{aligned}
$$

Note that $I_{\left(S_{u} \leq b^{T}(u)\right)} I_{\left(S_{u}=c\right)}=0, I_{\left(S_{u} \leq b^{T}(u)\right)} I_{\left(S_{u}<c\right)}=I_{\left(S_{u} \leq b^{T}(u)\right)}$. Therefore eventually we obtain

$$
C_{t}=\int_{0}^{t} c r(u) e^{-\int_{0}^{u} r(s) d s} I_{\left(S_{u} \leq b^{T}(u)\right)} d u .
$$

As we know, $Y_{t}=M_{t}-C_{t}, Y_{T}=M_{T}-C_{T}, Y_{T}=X_{T}$. Since

$$
Y_{t}-Y_{T}=Y_{t}-X_{T}=M_{t}-M_{T}+\left(C_{T}-C_{t}\right) \text {, }
$$

we have

$$
Y_{t}-\mathrm{E}\left(X_{T} \mid \mathcal{F}_{t}^{W}\right)=\mathrm{E}\left(C_{T}-C_{t} \mid \mathcal{F}_{t}^{W}\right)
$$


where $\left(\mathcal{F}_{t}^{W}\right)$ is the filtration generated by $W$. Multiplying both sides of equality

$$
e^{\int^{t} r(u) d u}, \text { we obtain }
$$

$$
\begin{aligned}
& V_{t}=e^{\int^{t} r(u) d u} \mathrm{E}\left(X_{T} \mid \mathcal{F}_{t}^{W}\right)+e^{\int^{t} r(u) d u} \mathrm{E}\left(C_{T}-C_{t} \mid \mathcal{F}_{t}^{W}\right), \\
& V_{t}=\mathrm{E}\left(e^{-\int_{t}^{T} r(u) d u}\left(c-S_{T}\right)^{+} \mid \mathcal{F}_{t}^{W}\right)+e^{\int^{t} r(u) d u} \mathrm{E}\left(C_{T}-C_{t} \mid \mathcal{F}_{t}^{W}\right), \\
& V_{t}=\mathrm{E}\left(e^{-\int_{t}^{T} r(u) d u}\left(c-S_{T}\right)^{+} \mid \mathcal{F}_{t}^{W}\right)+\mathrm{E}\left(\int_{t}^{T} c r(u) e^{-\int_{t}^{u} r(s) d s} I_{\left(S_{u} \leq b^{T}(u)\right)} d u \mid \mathcal{F}_{t}^{W}\right) .
\end{aligned}
$$

We know that $V_{t}=v^{T}\left(t, S_{t}\right)$, where $V_{t}$ is the value process of the American put option, and $v^{T}(s, x)$ is the value function.

Since $\mathcal{F}_{t}^{W}=\mathcal{F}_{t}^{S}, S_{t}(t, x)=x, x>0$, we have

$$
\begin{aligned}
v^{T}(t, x) & =\mathrm{E}\left(e^{-\int_{t}^{T} r(u) d u}\left(c-S_{T}(t, x)\right)^{+}\right) \\
& +\mathrm{E}\left(\int_{t}^{T} c r(u) e^{-\int_{t}^{u} r(s) d s} I_{\left(S_{u}(t, x) \leq b^{T}(u)\right)} d u\right), \\
v^{T}(t, x)= & \mathrm{E}\left(e^{-\int_{t}^{T} r(u) d u}\left(c-S_{T}(t, x)\right)^{+}\right) \\
+ & \int_{t}^{T} c r(u) e^{-\int_{t}^{u} r(s) d s} P\left(t, x ; u,\left(0, b^{T}(u)\right]\right) d u .
\end{aligned}
$$

Corollary 1. Let the functions $r(t)$ and $\sigma(t, x)$ be independent of the time, i.e., $r(t)=r, \sigma(t, x)=\sigma(x)$. Then for the American put option, the early exercise premium representation holds

$$
v^{T}(t, x)=\mathrm{E}\left(e^{-r(T-t)}\left(c-S_{T}(t, x)\right)^{+}\right)+\int_{t}^{T} c r e^{-r(u-t)} P\left(t, x ; u,\left(0, b^{T}(u)\right]\right) d u
$$

Proof. To prove this statement, it is sufficient to show that the boundary $b^{T}(t)$ is a monotone, in particular nondecreasing function, which automatically implies the fulfillment of the condition of Theorem 4.

We first show that the function $v^{T}(t, x)$ is nonincreasing with respect to $t$. Indeed, we have

$$
\begin{aligned}
& v^{T}(t, x)=\sup _{t \leq \tau \leq T} \mathrm{E}\left(e^{-r(\tau-t)}\left(c-S_{\tau}(t, x)\right)^{+}\right), \quad 0 \leq s<t \leq T, \\
& v^{T}(s, x)=\sup _{s \leq \tau \leq T} \mathrm{E}\left(e^{-r(\tau-s)}\left(c-S_{\tau}(s, x)\right)^{+}\right) .
\end{aligned}
$$


Since $S_{t}$ is a homogeneous Markov diffusion process, we have

$$
\begin{aligned}
& v^{T}(t, x)=\sup _{0 \leq \tau \leq T-t} \mathrm{E}\left(e^{-r \tau}\left(c-S_{\tau}(0, x)\right)^{+}\right), \\
& v^{T}(s, x)=\sup _{0 \leq \tau \leq T-s} \mathrm{E}\left(e^{-r \tau}\left(c-S_{\tau}(0, x)\right)^{+}\right) .
\end{aligned}
$$

From the latter equalities we find that $v^{T}(s, x) \geq v^{T}(t, x)$. We have

$$
v^{T}\left(s, b^{T}(t)+\varepsilon\right) \geq v^{T}\left(t, b^{T}(t)+\varepsilon\right)>g\left(b^{T}(t)+\varepsilon\right) .
$$

This inequality is true by virtue of the definition of the boundary. For each $\varepsilon>0$ we have $b^{T}(t)+\varepsilon \in D_{s}$ or, which is the same, $b^{T}(t)+\varepsilon>b^{T}(s)$. Finally, we obtain $b^{T}(s) \leq b^{T}(t)$, which in turn implies that the boundary $b^{T}(t)$ is a nondecreasing function.

Corollary 2. Let us consider the Black-Scholes model $r(t)=r, \sigma(t, x)=\sigma$. Then the value function of the American put option is written as

$$
\begin{aligned}
v^{T}(t, x) & =\mathrm{E}\left(e^{-r(T-t)}\left(c-S_{T}(t, x)\right)^{+}\right) \\
& +\int_{t}^{T} c r e^{-r(u-t)} \Phi\left(\frac{\ln \frac{b^{T}(u)}{x}-\left(r-\frac{\sigma^{2}}{2}\right)(u-t)}{\sigma \sqrt{u-t}}\right) d u .
\end{aligned}
$$

where $\Phi$ is the standard normal distribution function.

Proof. By Corollary 1, the value function of the American put option has the form

$$
v^{T}(t, x)=\mathrm{E}\left(e^{-r(T-t)}\left(c-S_{T}(t, x)\right)^{+}\right)+\int_{t}^{T} c r e^{-r(u-t)} P\left(t, x ; u,\left(0, b^{T}(u)\right]\right) d u .
$$

By virtue of the definition of transition probability we have

$$
P\left(t, x ; u,\left(0, b^{T}(u)\right]\right)=\mathrm{P}\left\{S_{u}(t, x) \leq b^{T}(u)\right\} .
$$

Using the condition $S_{t}=S_{0} \exp \left(\sigma W_{t}+\left(r-\frac{\sigma^{2}}{2}\right) t\right)$ we obtain

$$
\begin{aligned}
S_{u}(t, x) & =x \exp \left(\sigma\left(W_{u}-W_{t}\right)+\left(r-\frac{\sigma^{2}}{2}\right)(u-t)\right), \quad t \leq u \leq T, \\
P\left(t, x ; u,\left(0, b^{T}(u)\right]\right) & =\mathrm{P}\left\{\exp \left(\sigma\left(W_{u}-W_{t}\right)+\left(r-\frac{\sigma^{2}}{2}\right)(u-t)\right) \leq \frac{b^{T}(u)}{x}\right\} \\
& =\Phi\left(\frac{\frac{1}{\sigma} \ln \frac{b^{T}(u)}{x}-\frac{1}{\sigma}\left(r-\frac{\sigma^{2}}{2}\right)(u-t)}{\sqrt{u-t}}\right),
\end{aligned}
$$

i.e., we have

$$
\begin{aligned}
v^{T}(t, x) & =\mathrm{E}\left(e^{-r(\tau-t)}\left(c-S_{t}(t, x)\right)^{+}\right) \\
& +\int_{t}^{T} c r e^{-r(u-t)} \Phi\left(\frac{\ln \frac{b^{T}(u)}{x}-\left(r-\frac{\sigma^{2}}{2}\right)(u-t)}{\sigma \sqrt{u-t}}\right) d u .
\end{aligned}
$$




\section{ACKNOWLEDGEMENT}

The author expresses gratitude to Prof. M. Shashiashvili for the formulation of the problem and helpful hints. The author also extends thanks to the referee and Prof. N. Lazrieva whose careful reading of the manuscript has much contributed to its improvement.

\section{REFERENCES}

1. P. Babilua, Structure of an American type option value process in a general onedimensional diffusion model. Bull. Georgian Acad. Sci. 170(2004), 443-446.

2. C. Dellacherie and P.-A. Meyer, Probabilities and Potential. B. Theory of martingales. North-Holland Mathematics Studies, 72, North-Holland Publishing Co., Amsterdam, 1982.

3. S. D. JACKA, Optimal stopping and the American put. Math. Finance 1(1991), No. 2, $1-14$.

4. S. D. JACKA, Local times, optimal stopping and semimartingales. Ann. Probab. 21(1993), No. $1,329-339$.

5. N. El Karoui and I. Karatzas, A new approach to the Skorohod problem, and its applications. Stochastics Stochastics Rep. 34(1991), No. 1-2, 57-82.

6. N. V. Krylov, Controlled processes of diffusion type. (Russian) Nauka, Moscow, 1977.

7. R. S. Liptser and A. N. Shiryaev, Statistics of random processes. I. General theory. (Russian) Nauka, Moscow, 1974; English transl.: Applications of Mathematics (New York), 5. Stochastic Modelling and Applied Probability. Springer-Verlag, Berlin, 2001.

8. R. Myneni, The pricing of the American option. Ann. Appl. Probab. 2(1992), No. 1, $1-23$.

9. Ph. E. Protter, Stochastic integration and differential equations. 2nd ed. Applications of Mathematics (New York), 21. Stochastic Modelling and Applied Probability. SpringerVerlag, Berlin, 2004.

10. M. Shashiashvili, Semimartingale inequalities for Snell envelopes. Stochastics and Stochastics Rep. 43(1993), No. 1-2, 65-72.

11. A. N. Shiryaev, Yu. M. Kabanov, D. O. Kramkov, and A. V. Mel'nikov, Toward a theory of pricing options of European and American types. II. Continuous time. (Russian) Teor. Veroyatnost. i Primenen. 39(1994), No. 1, 80-129; English transl.: Theory Probab. Appl. 39(1994), No. 1, 61-102.

12. D. W. Strook and S. R. VAradhan, Multidimensional diffusion processes. Springer Verlag, Berlin, 1979.

13. M. Yor, Sur continuite des temps locaux associés a certaines semi-martingales. Société Mathématique de France. Astérisque 52-53(1978), 23-35.

(Received 12.09.2005)

Author's address:

Institute of Mechanics and Mathematics

Faculty of Exact and Natural Sciences

I. Javakhishvili Tbilisi State University

2, University St., Tbilisi 0143, Georgia

E-mail: p_babilua@yahoo.com 